(C) [2009] IEEE. Reprinted, with permission, from Huang, Yunkai; Zhu, Jianguo; Guo, Youguang. 2009, 'Thermal Analysis of High-Speed SMC Motor Based on Thermal Network and 3-D FEA with Rotational Core Loss Included', IEEE Transactions On Magnetics, Vol. 45, no. 10, pp. 4684-4687. This material is posted here with permission of the IEEE. Such permission of the IEEE does not in any way imply IEEE endorsement of any of the University of Technology, Sydney's products or services. Internal or personal use of this material is permitted. However, permission to reprint/republish this material for advertising or promotional purposes or for creating new collective works for resale or redistribution must be obtained from the IEEE by writing to pubs-permissions@ieee.org. By choosing to view this document, you agree to all provisions of the copyright laws protecting it. 


\title{
Thermal Analysis of High-Speed SMC Motor Based on Thermal Network and 3-D FEA with Rotational Core Loss Included
}

\author{
Yunkai Huang ${ }^{1}$, Jianguo Zhu², Senior Member, IEEE, Youguang Guo ${ }^{2}$, Senior Member, IEEE \\ ${ }^{1}$ School of Electrical Engineering, Southeast University, Nanjing, Jiangsu 210096, China \\ ${ }^{2}$ Faculty of Engineering, University of Technology, Sydney, NSW 2007, Australia
}

\begin{abstract}
This paper presents the thermal analysis of a highspeed motor with soft magnetic composite (SMC). Due to the high operation frequency, the core loss is much greater than the other losses and hence is the major heat source in the high-speed motor. Therefore it is of crucial importance to be able to calculate the core loss accurately. In this paper, the rotational core loss model is employed and implemented by using threedimensional (3-D) magnetic field analysis (FEA). Two methods to model the thermal behavior are presented. The first method uses a combination of lumped and distributed thermal parameters, which are obtained from motor dimensions and thermal constants. The second method employs 3-D FEA to accurately calculate the temperature distribution. Core losses are directly coupled into the thermal calculation by keeping the same hexahedral meshing for magnetic field analysis and thermal analysis. A testing bench for the high-speed SMC motor prototype has been set up to measure the core loss. The temperature rises were measured by thermal probes. The calculation and measurement results are compared and discussed.
\end{abstract}

Index Terms - Core loss, magnetic field finite element analysis (FEA), high-speed motor, lumped thermal parameters, soft magnetic composite (SMC) materials, thermal analysis

\section{INTRODUCTION}

High-speed motors are used in many applications, such as machine tool spindles, centrifugal compressors, vacuum pumps, friction welding units, and turbine generators. Compared with conventional general-purpose motors, the major advantages of high-speed motors are smaller size under a given power, higher power and torque densities, smaller moment of inertia and fast response, and directly connected with other high speed mechanical devices without gears [1, 2].

Due to the high operating frequency, the core loss is much greater than the other losses and hence is the major heat source. It has long been realized that a considerable amount of the total core loss in the stator core of a rotating electrical machine is caused by the rotating magnetic field [3]. Therefore, rotational core loss should be included for accurate calculation. Thermal analysis is one of the important issues in motor design, especially for high-speed motors. There are two basic analysis methods: the lumped parameter thermal model and the finite element method. Aglen [4] studied a high-speed generator using the lumped parameter thermal model to calculate the average temperatures at different parts of the motor, but the rotational core loss was not included in the model. Lamghari-Jamal et al. [5] used finite element method to calculate the temperature distribution of a high-speed synchronous reluctance machine, but the rotational core loss was not included either. Chun et al. [6] used the computational fluid dynamics method to analyze a high-speed generator, but they did not mention how to calculate the core loss.

This paper deals with the thermal behavior of the highspeed motor. The rotational core loss model is used and implemented by 3-D magnetic field analysis. Other losses, such as magnet loss and friction loss, are also carefully considered. Both the thermal network and FEA method are applied to compute the temperature distribution. The results are compared and discussed.

\section{SMC MOTOR PROTOTYPE}

To investigate the potential of SMC materials in manufacturing small motors of complex structures, a permanent magnet claw pole high-speed motor with an SMC core has been developed [7]. The major motor parameters are tabulated in Table I, and the structure (one pole pitch of one stack) is shown in Fig. 1.

TABLE I

MAIN DIMENSIONS AND DESIGN PARAMETERS

\begin{tabular}{cc}
\hline \hline Parameter and Dimension & Value \\
\hline Number of phases & 3 \\
Rated power $(\mathrm{W})$ & 2000 \\
Rated frequency $(\mathrm{Hz})$ & 666.7 \\
Rated speed $(\mathrm{rev} / \mathrm{min})$ & 20000 \\
Number of poles & 4 \\
Stator core material & SOMALOY ${ }^{\mathrm{TM}}$ 500 \\
Stator outer diameter $(\mathrm{mm})$ & 78 \\
Rotor core material & 29 \\
Rotor outer diameter $(\mathrm{mm})$ & 18 \\
Rotor inner diameter $(\mathrm{mm})$ & $\mathrm{NdFeB}$ \\
Permanent magnets & $75^{\circ}$ \\
Magnet arc & 2 \\
Magnet radial length $(\mathrm{mm})$ & 1 \\
Airgap length $(\mathrm{mm})$ & 48 \\
Axial length $(\mathrm{mm})$ & \\
\hline \hline
\end{tabular}




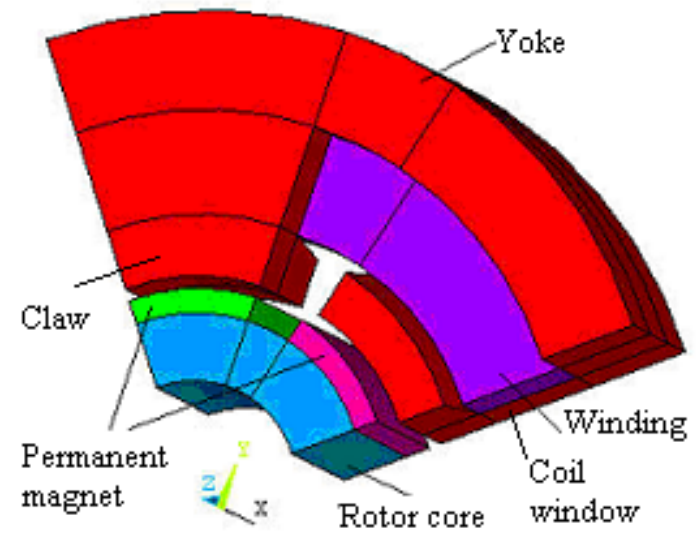

Fig. 1. Structure of a high-speed SMC claw pole motor (one pole pitch of one stack)

\section{HEAT SOURCES}

\section{A. Core loss}

Corresponding to various types of magnetizations and soft magnetic materials, a core loss model was developed, and has been applied to different types of electrical machines with verification by experimental results $[3,7,8]$.

In a soft magnetic material, the core loss with various typical magnetic flux patterns can be calculated by: Alternating flux:

$$
P_{a l t}=K_{a h} f B_{m}{ }^{h}+K_{a e}\left(f B_{m}\right)^{2}+K_{a a}\left(f B_{m}\right)^{3 / 2}
$$

Circularly rotating flux:

$$
P_{r o t}=P_{r h}+K_{r e}\left(f B_{m}\right)^{2}+K_{r a}\left(f B_{m}\right)^{3 / 2}
$$

where

$$
\frac{P_{r h}}{f}=a_{1}\left[\frac{1 / s}{\left(a_{2}+1 / s\right)^{2}+a_{3}^{2}}-\frac{1 /(2-s)}{\left[a_{2}+1 /(2-s)\right]^{2}+a_{3}^{2}}\right]
$$$$
\text { and } s=1-\frac{B_{m}}{B_{s}} \sqrt{1-\frac{1}{a_{2}^{2}+a_{3}^{2}}}
$$

$P_{r h}$ is the rotational hysteresis loss per cycle, and under elliptical rotating flux excitation:

$$
P_{\text {er }}=R_{B} P_{\text {rot }}+\left(1-R_{B}\right)^{2} P_{\text {alt }}
$$

where $R_{B}=B_{\text {min }} / B_{\text {maj }}$ is the axis ratio of the elliptical $\mathbf{B}$ locus.

By fitting (1)-(3) to the experimental results, the core loss coefficients in the above model were deduced as $K_{a h}=0.1402$, $h=1.548, K_{a e}=1.233 \mathrm{E}-5, K_{a a}=3.645 \mathrm{E}-4$ for alternating flux, and $K_{r e}=2.303 \mathrm{E}-4, K_{r a}=0, a_{1}=6.814, a_{2}=1.054, a_{3}=$ $1.445, B_{s}=2.134 \mathrm{~T}$ for circularly rotating flux.

In an electrical machine, the magnetic field distribution can be analyzed by the finite element method at various rotor positions and then the B locus in any element can be deduced. A series of elliptical loci can be obtained, when an arbitrary rotating flux density vector is expanded into a Fourier series. After determining the major and minor axes, $B_{k m a j}$ and $B_{k m i n}$, of the elliptical locus of the $k$-th harmonic flux density vector, the total loss in each element can be obtained by summing up the contributions from these harmonics, and the total core loss of the machine can then be obtained by summing up the core loss of each element as the following:

$$
P_{\text {core }}=\sum_{j=1}^{N_{e}} \sum_{k=0}^{\infty}\left[P_{\text {rotkj }} R_{B k j}+\left(1-R_{B k j}\right)^{2} P_{\text {altkj }}\right]
$$

where $P_{\text {rotkj }}$ and $P_{\text {altkj }}$ are the rotational and alternating core losses, respectively, and $R_{B k j}$ is the axis ratio of the elliptical locus of the $k$-th harmonic in the $j$-th element.

\section{B. Stator winding loss}

Global coil is used in the claw pole motor, as shown in Fig. 1. The copper loss consists of $I^{2} R$ loss and eddy current loss. As there is no end winding, the resistance can be calculated easily from the dimensions of coil window.

The eddy current loss is caused by (a) skin effect resulting from the same source conductors, and (b) proximity effect resulting from the motion of the permanent magnet. Since the multi-strand winding is used and the electrical frequency is low (about $700 \mathrm{~Hz}$ ), the skin depth is found to be larger than the radius of copper wire. Therefore, the skin effect can be ignored in this motor. By calculating the magnetic density in the winding at different rotor positions by FEA, it is found that the magnitude of flux density variation is very small, and hence the eddy current loss can be ignored.

\section{Magnet loss}

Analytical or FEA methods used in most literatures for calculating the rotor eddy current loss are based on 2-D magnetic field analysis, and the slotting effect is neglected. However, they cannot be used in the claw pole motor because the magnetic flux path is truly 3-D and it has large slot opening. In this paper, a dynamic circuit model described is used to calculate the eddy current loss in the permanent magnet [9]. The cross section of permanent magnet is divided into a number of slice pairs. Each slice pair forms an assumed eddy current loop. The slice thickness, $\Delta y$, is chosen such that within the slice the variation of eddy current density is almost linear. Inside the slice, therefore, the reaction of eddy currents on the magnetic field distribution can be ignored, and the flux density can be considered as uniformly distributed. The eddy current loss in the $k$ th slice pair is:

$$
P_{e k}=\left(\frac{2 l_{a} l_{r} \Delta y}{\sigma}\right) J_{k}{ }^{2}\left\{\frac{1}{3}\left[1+\left(1+\frac{J_{k-1}}{J_{k}}\right) \frac{J_{k-1}}{J_{k}}\right]\right\}
$$

where $l_{a}[\mathrm{~m}]$ and $l_{r}[\mathrm{~m}]$ are the magnet lengths in the axial and radial directions, respectively, $\sigma[1 / \Omega]$ is the conductivity of the magnet, $J_{k}\left[\mathrm{~A} / \mathrm{m}^{2}\right]$ and $J_{k-1}\left[\left[\mathrm{~A} / \mathrm{m}^{2}\right]\right.$ are the eddy current densities at the $k$-th and $(k-1)$-th slice pairs, respectively, and

$$
J_{k}=\sigma\left(\frac{d B_{r}}{d t}\right)_{k} \Delta y+J_{k-1}
$$

\section{Bearing loss}

The oil-lubricated rolling element bearings are used. The bearing loss is calculated according to the manufacturer manual [10].

\section{E. Air friction losses}

Because the ring magnets are mounted on the shaft, the surface of the rotor can be considered smooth. The air friction loss is calculated using the following equations [11]. 


$$
P_{a f}=C_{f} \pi \rho \omega^{3} r^{4} l
$$

where $\rho\left[\mathrm{Kg} / \mathrm{m}^{3}\right]$ is the density of air, $\omega[\mathrm{rad} / \mathrm{s}]$ is the angular velocity, $l[\mathrm{~m}]$ is the axial length and $r$ is the radius of the rotor. The friction coefficient $C_{f}$ is calculated by

$$
C_{f}=\frac{0.0152}{\operatorname{Re}_{\delta}^{0.24}}\left[1+\left(\frac{8}{7}\right)^{2}\left(\frac{4 \operatorname{Re}_{a}}{\operatorname{Re}_{\delta}}\right)^{2}\right]^{0.38}
$$

where $\operatorname{Re}_{\delta}=\frac{\rho \omega r \delta}{\mu}$ and $\operatorname{Re}_{a}=\frac{\rho v_{a} 2 \delta}{\mu}, \mu\left[\mathrm{N} . \mathrm{s} / \mathrm{m}^{2}\right]$ is the dynamic viscosity of air, $v_{a}[\mathrm{~m} / \mathrm{s}]$ is the peripheral speed of the rotor, and $\delta[\mathrm{m}]$ is the air gap length in the radial direction.

\section{THERMAL NETWORKS}

In general, the geometrical complexity of an electrical machine requires a large thermal network if a high resolution of temperature distribution is required. Instead of using a large, complex model, the geometrical symmetries of the machine were used to reduce the order of the model. The distributed thermal properties have been lumped together to form a small thermal network, representing the whole machine. For calculation of temperature distribution in the SMC motor, a thermal resistance network, as shown in Fig. 2, is used. It has ten nodes, and each node represents a specific part or region of the machine and the thermal resistances between the nodes include complex processes, such as twodimensional and three-dimensional heat flow, convection, internal heat generation, and variations in material properties. To account for all the three components of the heat flow at a node, the thermal structure is designed as in Fig. 3. As an example, the thermal resistances between the central node, $a$, and its adjacent nodes can be calculated by (9).

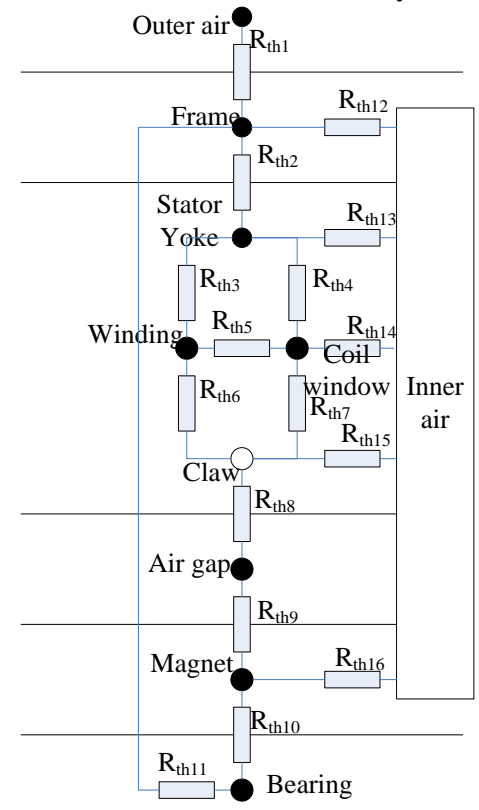

Fig. 2. Thermal network of one stack

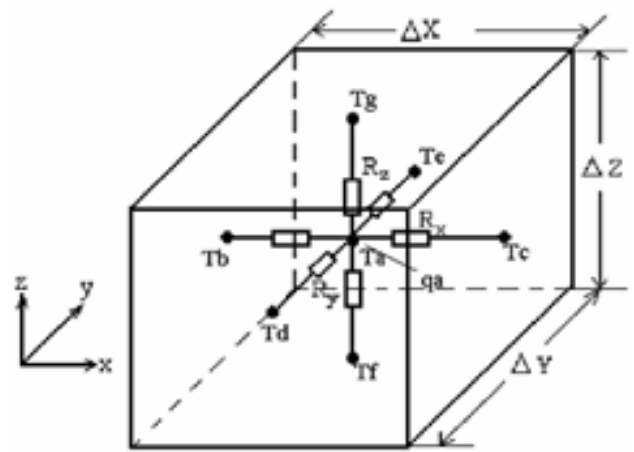

Fig. 3. Nodal thermal structure for 3-D heat flow

$R_{a b}=R_{a c}=R_{x}=\frac{\Delta X}{2 \lambda_{x} \Delta Y \Delta Z} \quad R_{a d}=R_{a e}=R_{y}=\frac{\Delta Y}{2 \lambda_{y} \Delta X \Delta Z}$

$R_{a f}=R_{a g}=R_{z}=\frac{\Delta Z}{2 \lambda_{z} \Delta X \Delta Y}$

After all the thermal resistances are determined and the power losses are allotted to appropriate nodes, the nodal temperatures rises in the steady-state can be solved by

$$
\Theta=\mathrm{G}^{-1} \mathrm{P}
$$

where $\Theta$ is the nodal temperature rise matrix, $\mathbf{P}$ is the nodal power loss matrix, and $\mathbf{G}$ is the inter-nodal conductance matrix, expressed by

$$
\mathrm{G}=\left[\begin{array}{cccc}
\sum_{i=1}^{n} \frac{1}{R_{1, i}} & -\frac{1}{R_{1,2}} & \cdots & -\frac{1}{R_{1, n}} \\
-\frac{1}{R_{2,1}} & \sum_{i=1}^{n} \frac{1}{R_{2, i}} & \cdots & -\frac{1}{R_{2, n}} \\
\vdots & \vdots & \ddots & \vdots \\
-\frac{1}{R_{n, 1}} & -\frac{1}{R_{n, 2}} & \cdots & \sum_{i=1}^{n} \frac{1}{R_{n, i}}
\end{array}\right]
$$

The calculation results at no-load (with an ambient temperature of $283 \mathrm{~K}$ ) are: $324.6 \mathrm{~K}$ for the frame, 326.3K for the stator yoke, $330.8 \mathrm{~K}$ for the coil window, $337.7 \mathrm{~K}$ for the claw pole, $334.4 \mathrm{~K}$ for the air gap, $331 \mathrm{~K}$ for the magnet, and $324.7 \mathrm{~K}$ for the bearing.

\section{THERMAL FIELD ANALYSIS}

In the thermal network, the core loss at each node cannot be obtained easily from the magnetic field calculation. In most cases, the average value is used. Because the core loss distribution is quite different in different positions of the stator core, 3-D FEA is used to analyze the temperature distribution in this paper. The heat transferred by radiation is ignored. The partial differential equation of the heat conduction and convection is expressed as

$$
\rho c\left(\frac{\partial T}{\partial t}+\{V\}^{T}\{L\} T\right)=\{L\}^{T}([D]\{L\} T)+Q
$$

where $\rho\left[\mathrm{kg} / \mathrm{m}^{3}\right]$ is the mass density, $c[\mathrm{~J} /(\mathrm{kgK})]$ is the specific heat, $\mathrm{T}[\mathrm{K}]$ is the temperature, $\mathrm{t}[\mathrm{s}]$ is the time, $\{L\}$ is the vector operator, $\{V\}$ is the velocity vector for mass transport of heat, $[D]$ is the conductivity matrix, $K_{x x}, K_{y y}$ and $K_{z z}[\mathrm{~W} /(\mathrm{m} \cdot \mathrm{K})]$ are the thermal conductivity alone the $\mathrm{X}, \mathrm{Y}$ 
and $\mathrm{Z}$ axes, respectively, and $Q\left[\mathrm{~W} / \mathrm{m}^{3}\right]$ is the heat generation rate per unit volume. For steady-state analysis, the first term on left-hand side of (12) is zero.

The core loss in each element obtained from the magnetic field solutions is applied as a body load to the corresponding element in thermal analysis by keeping the same element meshing. The boundary conditions can be of Dirichlet type, where the temperature $T^{*}$ on the boundary is specified. This boundary condition can be applied to the surface between the frame and outer air. A Neumann type of boundary condition specifies the heat flux $q_{n}$ through the boundary. The Newton's convection boundary condition is:

$$
q_{n}=n \bullet(k \nabla T)=\alpha\left(T-T_{a m b}\right)
$$

The heat flux flow through the boundaries to the surrounding is described with the heat transfer coefficient $\alpha$ and the external temperature $T_{a m b}$. The convective heat transfer to the surroundings is dependent on the geometry and the cooling conditions. Air gap convection coefficient is determined by two main quantities: the ruggedness of the rotor and stator surfaces and the peripheral speed of the rotor surface. By assuming a smooth surface, the convection coefficient can be calculated by the experiential formula [11]

$$
\begin{gathered}
h_{\delta}=28\left(1+\omega_{\delta}{ }^{0.5}\right) \\
\omega_{\delta}=\sqrt{\left(0.5 v_{r}\right)^{2}+v_{a}{ }^{2}}
\end{gathered}
$$

where $v_{r}$ and $v_{a}$ are the peripheral and axial velocities of the rotor surface, respectively. Other losses, such as the copper loss and air friction loss, are also applied to the model as body loads. The temperature distribution at no-load is shown in Fig. 4. The temperature is mainly affected by the core loss, which that in the claw poles is greater than that in other parts because the magnitude of $\mathrm{B}$ variation in the claws is greater.

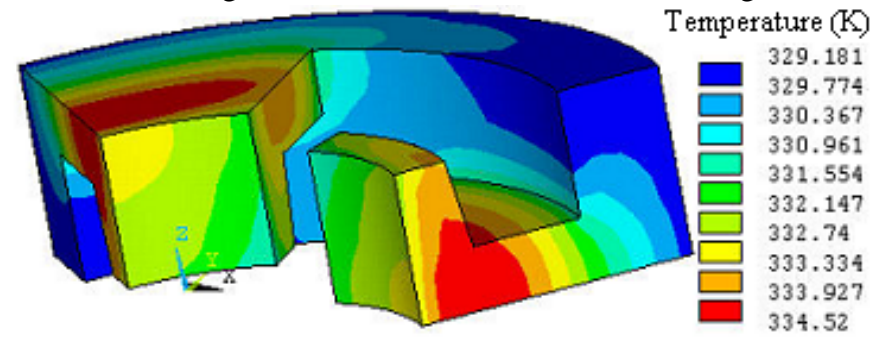

Fig. 4. Temperature distribution of stator core

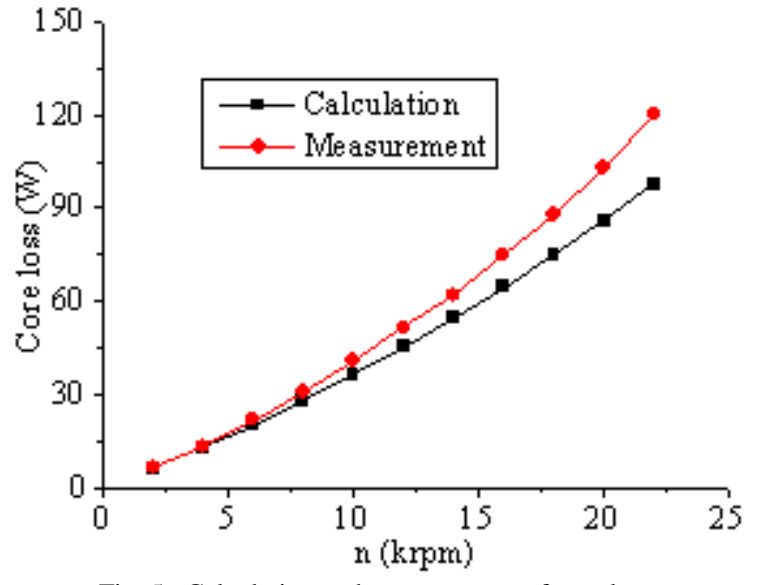

Fig. 5. Calculation and measurement of core loss

\section{EXPERIMENTAL RESULTS}

A testing bench has been set up to measure the core loss and temperature of the motor prototype. The core loss is measured at no-load by the dummy rotor method. The calculated and measured core losses are compared in Fig. 5. Below $12 \mathrm{krpm}$, the error is less than $10 \%$, but the maximum error is about $15 \%$ when the speed is over $12 \mathrm{krpm}$. The possible reasons are: (1) loss coefficients derived from the rotational core loss data at low frequency have some errors when they are used in high frequency; (2) rotor loss cannot be distinguished from the measurement results.

The temperature is measured by infrared temperature probe. At $20 \mathrm{krpm}$ and no-load, the frame temperature is $331.4 \mathrm{~K}$, the stator yoke temperature is $333.5 \mathrm{~K}$, and the coil temperature is $334.2 \mathrm{~K}$. The measured temperatures are higher than the calculations, because the actual loss is greater than the calculation.

\section{CONCLUSION}

The thermal behavior of high-speed SMC motor is studied in this paper. The thermal network and 3-D FEA methods are used and compared. The core loss, the major heat source in the high speed claw pole motor, is calculated by rotational core loss model, and is directly coupled into 3-D thermal field analysis. As a result, the accurate temperature distribution can be acquired. The analysis methods have been validated by the measurements on the motor prototype.

\section{REFERENCES}

[1] M. Johansson, M. Larsson, L. Naslund, and J. Hylander, "Small highspeed induction motors," in Proc. IEEE Int. Electric Machines and Drives Conf., vol. 1, June 2003, pp. 279-284.

[2] N. Bianchi, S. Bolognani, and F. Luise, "Potentials and limits of highspeed PM motors," IEEE Trans. on Industry Applications, vol. 40, pp. 1570-1578, 2004.

[3] J. G. Zhu and V. S. Ramsden, "Improved formulations for rotational core losses in rotating electrical machines," IEEE Trans. Magn., vol. 34, pp. 2234-2242, July 1998.

[4] O. Aglen and A. Andersson, "Thermal analysis of a high-speed generator," in Proc. IEEE Int. Electric Machines and Drives Conf., vol. 1, June 2003, pp. 547-554.

[5] M. I. Lamghari-Jamal, J. Fouladgar, E. H. Zaim, and D. Trichet, “A magneto-thermal study of a high-speed synchronous reluctance machine,” IEEE Trans. Magn., vol. 42, pp. 1271-1274, 2006.

[6] Y. Chun, K. Y. Wei, and X. N. Wang, "Coupled-field thermal analysis of high-speed permanent magnetic generator applied in micro-turbine generator," in Proc. $8^{\text {th }}$ Int. Conf. on Electrical Machines and Systems, 2005, vol. 3, pp. 2458-2461.

[7] Y. K. Huang, J. G. Zhu, Y. G. Guo, and Q. S. Hu, "Design and analysis of a high-speed claw pole motor with soft magnetic composite core," IEEE Trans. Magn., vol. 43, no. 6, pp. 2492 - 2494, June 2007.

[8] Y. G. Guo, J. G. Zhu, J. J. Zhong, and W. Wu, "Core losses in claw pole permanent magnet machines with soft magnetic composite stators," IEEE Trans. Magn., vol. 39, pp. 3199-3201, 2003.

[9] J. G. Zhu, S. Y. R. Hui, and V. S. Ramsden, "A generalized dynamic circuit model of magnetic cores for low- and high-frequency applications. I. Theoretical calculation of the equivalent core loss resistance," IEEE Trans. Power Electronics, vol. 11, pp. 246-250, March 1996.

[10]SKF, SKF Bearing Manual, Oct. 2004.

[11]J. Saari, Thermal Analysis of High-Speed Induction Machines, PhD Thesis, Electrical Engineering, Helsinki University of Technology, Finland, 1998, p. 73. 
